\title{
Evolution of Buoyant Bubbles in M87
}

\author{
E.Churazov ${ }^{1,2}$, M.Brüggen ${ }^{1,3}$, C.R.Kaiser ${ }^{1}$, H.Böhringer ${ }^{4}$, W.Forman ${ }^{5}$
}

\begin{abstract}
The morphology of the X-ray and radio emitting features in the central $\sim 50 \mathrm{kpc}$ region around the galaxy M87 strongly suggests that buoyant bubbles of cosmic rays (inflated by an earlier nuclear active phase of the galaxy) rise through the cooling gas at roughly half the sound speed. In the absence of strong surface tension, initially spherical bubbles will transform into tori as they rise through an external medium. Such structures can be identified in the radio images of the halo of M87. During their rise, bubbles will uplift relatively cool X-ray emitting gas from the central regions of the cooling flow to larger distances. This gas is colder than the ambient gas and has a higher volume emissivity. As a result, rising "radio" bubbles may be trailed by elongated $\mathrm{X}$-ray features as indeed is observed in M87. We performed simple hydrodynamic simulations to qualitatively illustrate the evolution of buoyant bubbles in the M87 environment.
\end{abstract}

Subject headings: galaxies: active - galaxies: clusters: individual: Virgo - cooling flows - galaxies: individual: M87 - X-rays: galaxies

\section{Introduction}

The giant elliptical galaxy M87 continues to be the subject of numerous experimental and theoretical studies. It is located at, or near, the center of the very nearby $(\sim 18 \mathrm{Mpc}, 1$ arcminute corresponds to $\sim 5 \mathrm{kpc}$ ) irregular Virgo cluster and is at the center of a weak cooling flow with an estimated mass deposition rate of some 10-40 $M_{\odot}$ per year (e.g. Peres et al. 1998). The radio source Virgo A (3C274), associated with the galaxy M87, is well known because of the spectacular jet which is observed both in radio and optical bands (see e.g. Biretta 1999 for a review). In addition to the jet, which is contained within the central $2 \mathrm{kpc}$ or $30^{\prime \prime}$ region, there is a lower surface brightness radio halo extending up to a distance of $\sim 40-50$

\footnotetext{
${ }^{1}$ MPI für Astrophysik, Karl-Schwarzschild-Strasse 1, 85740 Garching, Germany

${ }^{2}$ Space Research Institute (IKI), Profsoyuznaya 84/32, Moscow 117810, Russia

${ }^{3}$ Churchill College, Storey's Way, Cambridge CB3 0DS, United Kingdom

${ }^{4}$ MPI für Extraterrestrische Physik, P.O.Box 1603, 85740 Garching, Germany

${ }^{5}$ Harvard-Smithsonian Center for Astrophysics, 60 Garden St., Cambridge, MA 02138
}

kpc from the nucleus (e.g. Kassim et al. 1993, Böhringer et al. 1995, Owen, Eilek and Kassim 1999, 2000).

The $\mathrm{X}$-ray emission is strongly peaked at the position of the nucleus of M87 and is largely symmetric around it. However, it was found in the Einstein HRI observations (Feigelson et al. 1987) and then in the ROSAT data (Böhringer at al. 1995) that there are departures from a symmetric model and these departures resemble the morphology of some prominent features in the outer radio lobes. These findings initiated numerous discussions on the interaction of the radio and $\mathrm{X}$ ray emitting plasmas (e.g. Feigelson et al. 1987, Böhringer et al. 1995, Böhringer et al. 1999, Owen, Eilek and Kassim 1999,2000, Harris et al. 1999). In a number of other cooling flow clusters with strong radio halos surrounding the dominant galaxy (e.g. Perseus - Böhringer at al. 1993, McNamara et al. 1996, Churazov et al. 2000, Fabian et al. 2000a, A4059 - Huang and Sarazin 1998, Hydra A - McNamara et al. 2000) there is a clear anticorrelation of the radio and $\mathrm{X}$-ray emitting plasmas. This makes the case of M87 especially interesting.

We discuss below one particular model which is 
able to reproduce qualitatively both the radio and $\mathrm{X}$-ray morphologies observed in the central region surrounding the M87 galaxy.

\subsection{Radio and $X$-ray morphology}

There is a long history of M87 radio halo observations, starting with Mills (1952) shortly after the initial discovery of the radio source Virgo A (Bolton, Stanley and Slee 1949). Because of the very bright compact source, the dynamic range of the images was always a problem. Recently, Owen et al. (2000) presented a new high resolution, high dynamic range map of the halo of M87 at $327 \mathrm{MHz}$ (see Figure 1). For the sake of further discussion this map has been rotated by 90 degrees to have East up and North to the right. In this map several distinct structures are discernible. The high brightness centre represents the well-studied inner lobe structure (oriented approximately east-west) with the famous jet pointing west north-west (approximately bottom-right for the orientation of images adopted in Fig.1). The highly structured outer halo is much fainter. It consists of the ear-shaped (torus-like) eastern bubble, the much less well-defined western bubble, both of which are connected to the central emission by a 'trunk', and the two very faint, almost circular, emission regions northeast and southwest of the centre.

Owen et al. (2000) find a peak surface brightness in the region of the eastern bubble of about $53 \mathrm{mJy} /$ beam while the circular regions range from $14 \mathrm{mJy} /$ beam to about $25 \mathrm{mJy} /$ beam. Rottmann et al. (1996) show that all of the described structures are also present at $10.6 \mathrm{GHz}$. They also show that the outer halos have a radio spectral index in the range from -2.8 to -2.5 between $4.8 \mathrm{GHz}$ and $10.6 \mathrm{GHz}$. The spectral index is flatter by a factor of about 1.5 between $330 \mathrm{MHz}$ and $1.5 \mathrm{GHz}$. This suggests that radiative energy losses of the synchrotron emitting particles lead to a significant steepening of the radio spectrum at about $3 \mathrm{GHz}$. Unfortunately, the maps used by Rottmann et al. (1996) have low resolution since they are obtained with single dish observations and so the spectral index distribution of the halo and its connection to the detailed structures, e.g., bubbles and attached "trunks", is not known.

In Fig.1 (lower left) we show the $\mathrm{X}$-ray surface brightness distribution of the same region (and similar orientation) as the radio map. The data are the combined $200 \mathrm{ksec}$ ROSAT/HRI image, adaptively smoothed to suppress high frequency noise. As pointed out by a number of authors (Feigelson et al. 1987, Böhringer at al. 1995, Owen, Eilek and Kassim 1999, Harris et al. 1999) there is evidence for a correlation between $\mathrm{X}$-ray and radio emitting features. The simplest explanation of this correlation is that the excess $\mathrm{X}$-ray emission is due to inverse Compton scattering of the cosmic microwave background photons by the same relativistic electrons which produce the synchrotron radio emission (Feigelson et al. 1987). However, ROSAT/PSPC observations have shown that the excess emission has a thermal spectrum (Böhringer at al. 1995) and the X-ray emitting gas in these region has a lower temperature than that in the ambient regions.

Thus, we summarize three observational facts which we are trying to explain below:

- There are prominent "torus-like" features in the radio image.

- There is a correlation (but not one to one) of the $\mathrm{X}$-ray and radio bright regions.

- Excess X-ray emission, associated with the radio bubbles and attached "trunks", arises from thermal gas whose temperature is less than that of the ambient X-ray emitting gas.

\subsection{Analogy with atmospheric explosions}

Concentrating on the "torus-like" radio features one can note its striking similarity with some evolutionary stages of hot buoyant bubbles formed by a powerful (e.g. nuclear) explosion in the Earth's atmosphere. The transformation of the initially spherical bubble into a torus is a common and well known property of buoyant bubbles lacking strong surface tension (e.g. Walters and Davison 1963, Onufriev 1967, Zhidov et al. 1977). Morphologically similar structures resembling "mushrooms" appear in Rayleigh-Taylor unstable configurations: as the fluid rises through the ambient medium, Kelvin-Helmholtz instabilities create the torus-like head of the "mushroom". Images of various laboratory experiments where such structures were observed and discussion of the relevant physical mechanisms are widespread 

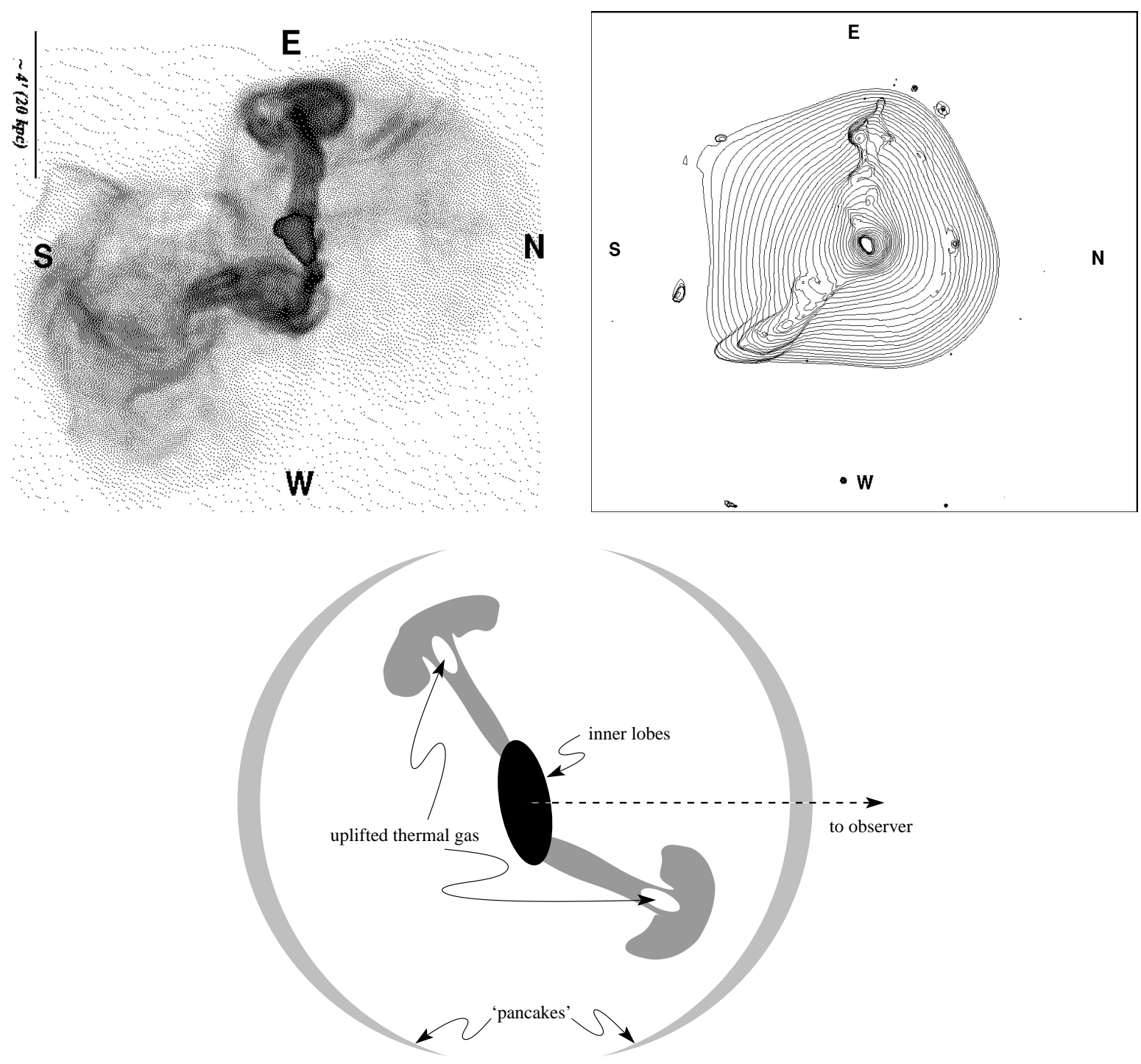

Fig. 1.- Top-Left: $14^{\prime} .6 \times 16^{\prime} .0$ map of the radio halo of M87 at $327 \mathrm{MHz}$ rotated $90^{\circ}$ clockwise (North is to the right, East is up). The map was kindly provided by F.Owen (see Owen, Eilek and Kassim 2000 for original data). Top-Right: Adaptively smoothed ROSAT HRI X-ray image. The size and orientation of the image are the same as for the radio image. Bottom: Possible geometry of the source inspired by analogy with "mushroom clouds" produced by powerful atmospheric explosions. The black region in the center denotes the inner radio lobes, gray "mushrooms" correspond to the buoyant bubbles already transformed into tori, and the gray lens-shaped structures are the 'pancakes' formed by the older bubbles. To explain the observed morphology, the source must be oriented close to the line of sight (dashed line). The pancakes are shown edge-on as shaded regions. 
(see e.g. Batchelor 1967, Turner 1973, Inogamov 1999). The idea that buoyancy plays an important role in the evolution of the radio lobes in galaxies was first proposed by Gull and Northover (1973) and has been used to estimate the life time of the radio lobes in M87 by Böhringer et al. (1995). The role of buoyancy was also discussed by Baum and O'Dea (1991) in application to PKS 0745-191. The similarity of the M87 "torus-like" features to the Rayleigh-Taylor mushrooms was pointed out by Churazov et al. (2000) and to a subsonic vortex ring by Owen, Eilek and Kassim (2000).

Further pursuing the analogy with powerful explosions, we note that during the transformation of a bubble into a torus some ambient gas is captured and uplifted by the rising bubble/torus. In the cooling flow surrounding M87, the entropy of the $\mathrm{X}$-ray emitting gas rises quickly with distance from the centre, and therefore the emissivity of the gas increases very steeply if it remains in pressure balance with the ambient gas (Böhringer et al. 1995, Nulsen 1997). This may qualitatively explain the correlation of the radio and $\mathrm{X}_{-}$ ray emitting plasmas and naturally accounts for the thermal nature of the excess thermal emission.

In the case of an atmospheric explosion, the final stage of the hot bubble occurs when the bubble reaches a height at which the density of the ambient gas is equal to the density of the bubble. Then (neglecting oscillations near the equilibrium position) the bubble expands laterally (forming a "pancake") to occupy a thin layer in the atmosphere having the same density. The bubbles/tori in M87 may share the same fate. In a spherically symmetric potential, the bubble will try to fill a segment (equipotential surface) of a sphere. The largest distinct features in the radio map (see Fig.1) could be just those late-stage bubbles.

The sketch of the possible overall source structure based on the analogy with a powerful atmospheric explosion is shown in Fig.1. Here the black region in the center denotes the inner radio lobes, the gray mushrooms correspond to the buoyant bubbles already transformed into tori and the gray lens-shaped structures are the "pancakes" formed by the older bubbles.

Buoyant bubbles and their role in the complex $\mathrm{X}$-ray and radio morphology of M87 have been discussed previously e.g. in Böhringer et al. (1995),
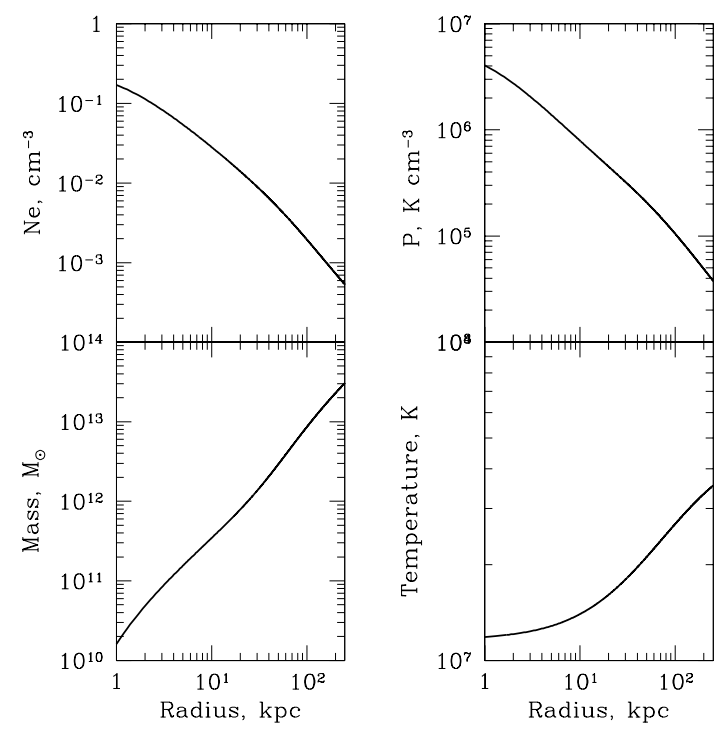

Fig. 2.- Initial distribution of electron density, temperature, pressure and gravitating mass (adopted from Nulsen and Böhringer 1995) assumed in the simulations.

Nulsen (1997), Böhringer (1999), Owen, Eilek and Kassim (2000). Below we follow the line of arguments suggested in Churazov et al. (2000) and present hydrodynamic calculations of the radio lobe evolution to illustrate the qualitative picture described above.

\section{Method}

In the next sections we describe the method, initial conditions, and assumptions used in the numerical simulations of bubbles in a hot gaseous atmosphere. We first detail the method and the use of "tracer" particles to track the motion of hot gas which is entrained in the rising bubble. We next describe our approach to calculation of the synchrotron radio emission from the evolving bubble.

\subsection{Hydrodynamic simulations}

The simulations were obtained using the ZEUS-3D code which was developed especially for problems in astrophysical hydrodynamics (Stone \& Norman 1992a). The code uses finite differencing on an Eulerian grid and is fully 
explicit in time. It is based on an operator-split scheme with piecewise linear functions for the fundamental variables. The fluid is advected through a mesh using the upwind, monotonic interpolation scheme of van Leer (1977). For a detailed description of the algorithms and their numerical implementation see Stone \& Norman (1992a, b). To study the spectral aging of the relativistic gas, the ZEUS code was modified to follow the motion of 'tracer' particles which are advected with the fluid.

In our simulations we employed an ideal gas equation of state and we ignored the effects of magnetic fields and rotation. The cooling time is sufficiently long compared to the time scales considered here since, for an electron density of $0.02 \mathrm{~cm}^{-3}$ and temperature of the order of $2 \mathrm{keV}$ (see Fig.2) the cooling time is $\sim 5 \times 10^{8}$ years. This is an order of magnitude longer than the typical duration of the run of $\sim 5 \times 10^{7}$ years. We therefore completely neglected cooling. The simulations were computed on a spherical grid in two dimensions and were performed on an IBM RS/6000 cluster. The computational domain spans $51 \mathrm{kpc}$ in radius and $\pi / 2 \mathrm{rad}$ in angle and was covered by $200 \times 200$ grid points (in the $r$ and $\theta$-direction).

A model for the mass and initial temperature was adopted from Nulsen \& Böhringer (1995) which is shown in Fig.2. The gas density distribution was then found by assuming hydrostatic equilibrium to maintain an initially static model. A spherical bubble was created with a radius of $r_{\mathrm{b}}=5 \mathrm{kpc}$ at a distance of $d=9 \mathrm{kpc}$ from the gravitational center. It was made buoyant by reducing its density with respect to the background by a factor of 100 and simultaneously raising the temperature by the same factor. During its subsequent evolution, the gas is treated as a single fluid which is assumed to obey a polytropic equation of state with $\Gamma=5 / 3$. The bubble was filled with 2000 tracer particles and was placed on the $\theta=0$ axis along which it rose in the gravitational potential. Subsequently, we rotated the two-dimensional output about this axis (azimuthally). To test the assumption of rotational symmetry, we performed some 3D simulations with reduced resolution and found that this assumption is fairly robust as far as the global motions and morphologies are concerned. As we discuss in detail below, buoyancy deforms the bubble and drives it through the ambient medium as shown in Fig.3. Shear instabilities cause the formation of tori which separate from the main bubble. This process, also called "vortex shedding", has been observed and studied extensively (see Norman et al. 1982).

Finally, we should address some issues related to the accuracy of these kinds of finite-difference hydrodynamic simulations. While the code can simulate large-scale mixing due to Rayleigh-Taylor and Kelvin-Helmholtz instabilities, it does not include real particle diffusion. Any observed diffusion is therefore entirely numerical. The boundary between the bubble and the ambient medium becomes less sharp as the simulation proceeds due to discretization errors in the advection scheme. For a test of the advection algorithm in the ZEUS code see Stone \& Norman (1992a,b). In simple advection tests, it was found that, during the advection of a sharp discontinuity over a grid of 200 zones, the discontinuity is spread over 3-4 grid cells. Therefore, the small features in our simulation are likely to be affected by these advection errors whereas the larger features are not.

Second, numerical viscosity is also responsible for suppressing small-scale instabilities at the interface between the bubble and the cooler, surrounding, X-ray emitting gas. To assess the effects of numerical viscosity, we have repeated our simulations on grids with $100 \times 100$ grid points. From our experiments, we can conclude that "global parameters" such as the position and size of the bubble as well as the presence of "toroidal" structure are relatively insensitive to the resolution. The detailed small scale morphology does depend on the resolution and the initial conditions.

Finally, we stress that the simulations presented here are not intended to provide a detailed simulation of the radio/X-ray structure surrounding M87. Rather they are intended as a guide, a toy model, to assist our interpretation of the complex emission (see discussion in Section 3.1). 


\subsection{Simulation of the radio emission}

The observations of radio synchrotron emission from the buoyant bubbles in M87 imply the presence of a relativistic, magnetized plasma. However, the simulations presented in previous sections are based on a purely hydrodynamic scheme with a single, non-relativistic fluid. To estimate the radio flux expected from the simulation, we make the following assumptions.

The relativistic plasma and the associated magnetic field, the 'relativistic fluid', are confined within small volumina intermixed with the nonrelativistic, thermal plasma governing the dynamics. This implies that the relativistic fluid may mix with the thermal fluid on macroscopic scales but not on microscopic ones. This is very similar to the behavior of air bubbles in water which are confined by surface tension (e.g. Zhidov et al. 1977).

We assume the magnetic field to be tangled on scales small compared to the size of the volumina of relativistic fluid. This allows us to treat the magnetic field as part of the relativistic fluid. The total energy density inside the small volumina is therefore the sum of the energy density of the magnetic field, $u_{\mathrm{B}}$, and that of the relativistic particles, $u_{\mathrm{e}}$. We assume that the small volumina of relativistic fluid are in pressure equilibrium with the thermal fluid throughout the simulation, i.e. $3 p_{\text {th }}=u_{\mathrm{B}}+u_{\mathrm{e}}$. We also assume that there is no significant re-acceleration of relativistic particles in the bubble during its evolution.

At the start of the simulation, we assume some initial value of the magnetic field (the same value for every small volumina of relativistic particles within the bubble) and a power law distribution for the relativistic electrons (in terms of the particle Lorentz factor), $\gamma, n_{\mathrm{e}}\left(t_{\mathrm{o}}\right) d \gamma=n_{\mathrm{o}} \gamma^{-p} d \gamma$. At the start of the simulation $u_{\mathrm{e}}$ is therefore given by

$$
u_{\mathrm{e}}=m_{\mathrm{e}} c^{2} n_{\mathrm{o}} \int_{\gamma_{\min }}^{\gamma_{\max }} \gamma^{-p}(\gamma-1) d \gamma
$$

where we assume $\gamma_{\min }=1, \gamma_{\max }=10^{6}$. For a given initial magnetic field the normalization $n_{\mathrm{o}}$ of the particle distribution is then adjusted such that the entire relativistic fluid is in pressure equilibrium with the thermal fluid.

For values of $p \geq 2$, the energy density of relativistic particles is dominated by low energy par- ticles. The life time of such particles, due to radiative losses, is much longer than the duration of the simulation (and no Coulomb losses are present since we assume that thermal gas is not mixed with relativistic particles on microscopic scales). The motion of the bubble (see Section 3) is found to be subsonic even with respect to the cluster gas. We therefore assume that the energy density of the magnetic filed and relativistic particles changes adiabatically according to the changes of the pressure of the surrounding thermal gas: $u_{\mathrm{B}} \propto u_{\mathrm{e}} \propto p_{\text {th }}$. Note, however, that our hydrodynamic simulations assume an adiabatic index of $5 / 3$ for the nonrelativistic gas, while the relativistic fluid expands and contracts adiabatically with an adiabatic index $\Gamma_{\mathrm{r}}=4 / 3$. This implies that, during the expansion of a buoyant bubble, the fraction of the volume occupied by the relativistic fluid increases relative to the volume of the thermal fluid as $V_{\mathrm{r}} \propto V_{\mathrm{th}}^{5 / 4}$. Strictly speaking this means that accounting for relativistic fluid with a different adiabatic index will not affect the dynamics of the bubble evolution as long as the fraction of the volume occupied by the relativistic fluid is small. We will see in the following that this assumption is probably invalid, but we believe that it does not very severely affect the results.

The energy losses of the relativistic particles are due to adiabatic expansion, synchrotron radiation and inverse Compton scattering of the CMB. These losses depend on the pressure history of each volumina of relativistic particles. The pressure history can be recovered using the tracer particles. For a given region within the bubble the change of the Lorentz factor $\gamma$ of a given electron due to adiabatic expansion or contraction of the relativistic fluid and radiative losses can be written as

$$
\begin{aligned}
\frac{d \gamma}{d t} & =a \frac{\gamma}{p_{\mathrm{th}}(t)} \frac{d p_{\mathrm{th}}(t)}{d t} \\
& -\frac{4 \sigma_{\mathrm{T}}}{3 m_{\mathrm{e}} c} \gamma^{2}\left[u_{\mathrm{B}}\left(t_{\mathrm{o}}\right) \frac{p_{\mathrm{th}}(t)}{p_{\mathrm{th}}\left(t_{\mathrm{o}}\right)}+u_{\mathrm{IC}}\right]
\end{aligned}
$$

where $a=(\Gamma-1) / \Gamma=1 / 4$.

This can be integrated (e.g. Kaiser, DennettThorpe \& Alexander 1997) to give the relation between the Lorentz factor of the electron at time $t$ and the initial Lorentz factor of the same electron 


$$
\gamma_{0}=\frac{\gamma p_{\mathrm{th}}(t)^{a}}{p_{\mathrm{th}}\left(t_{0}\right)^{a}+\gamma b}
$$

with

$b=\frac{4 \sigma_{\mathrm{T}}}{3 m_{\mathrm{e}} c} \int_{t_{\mathrm{o}}}^{t}\left[u_{\mathrm{B}}\left(t_{\mathrm{o}}\right) \frac{p_{\mathrm{th}}(t)}{p_{\mathrm{th}}\left(t_{\mathrm{o}}\right)}+u_{\mathrm{IC}}\right] p_{\mathrm{th}}(t)^{a} d t$.

The value of $b$ is calculated from the pressure history of the tracer particles. The integral in this expression must be evaluated numerically using the discrete time steps of the simulation.

Finally, the adiabatic change of the volume in which the relativistic particles are contained implies $n_{\mathrm{o}}(t)=n_{\mathrm{o}}\left(t_{\mathrm{o}}\right)\left[p_{\mathrm{th}}(t) / p_{\mathrm{th}}\left(t_{\mathrm{o}}\right)\right]^{1 / \Gamma}$. The energy distribution of relativistic electrons at time $t$ is then

$$
n_{\mathrm{e}} d \gamma=n_{\mathrm{o}}(t) \gamma_{0}^{-p} \frac{d \gamma_{0}}{d \gamma} d \gamma=n_{\mathrm{o}}\left(t_{\mathrm{o}}\right) \frac{p_{\mathrm{th}}(t) \gamma_{\mathrm{o}}^{2-p}}{p_{\mathrm{th}}\left(t_{\mathrm{o}}\right) \gamma^{2}} d \gamma
$$

The energy distribution of the relativistic particles combined with the strength of the magnetic field yields the monochromatic synchrotron emissivity (e.g. Longair 1994)

$$
j_{\nu}=\frac{4}{3} \sigma_{\mathrm{T}} c u_{\mathrm{B}} \int_{\gamma_{\min }}^{\gamma_{\max }} \gamma^{2} \phi(\gamma, \nu) n_{\mathrm{e}} d \gamma
$$

where $\phi(\gamma, \nu)$ is the dimensionless spectrum emitted by a single electron.

To obtain the synchrotron surface brightness from the simulation, we assume rotational symmetry about the polar-axis and integrate the emissivity along lines of sight through the resulting 3dimensional model.

\section{Results and discussion}

With the foundation and assumptions described in the previous section, we describe the results of two numerical simulations. We first present the results from the two numerical simulations with different initial conditions for the bubbles. We compute the velocity and position versus time of the rising bubble. For the first simulation, we compute the synchrotron radio emission from the bubble for two orientations, one aligned with the symmetry axis in the plane of the sky and the second withthe symmetry axis aligned $45^{\circ}$ with respect to the plane of the sky. The two orientations differ in the initial conditions for the magnetic field strength in order to reproduce the observed radio spectrum. We derive the X-ray appearance of the bubble from the first simulation, also for the two different orientations. Finally, we discuss the "pancake" phase and calculate the largest distance to which bubbles can rise as a function of their initial location and volume fraction of trapped, hot gas.

\subsection{Bubble configuration}

Fig.3 shows snapshots of the temperature distribution during the evolution of the bubble. One can identify two stages of the bubble evolution. The initially spherical bubble flattens and develops a "cone" at the rear, which is filled with entrained gas. The bubble then transforms into a torus which at later stages may fragment into smaller structures, but which continue to show a global toroidal structure. Note that ambient gas (in particular the gas captured during the bubble $\rightarrow$ torus transformation) occupies the central part of the rising structure. Note also that in the last stages (shown in Fig.3) the coldest temperatures are found not at the center of the cluster but within the rising structure. We stress again that no radiative cooling was included in the simulations and these low temperatures regions are associated with gas which has been uplifted from the central regions and has expanded adiabatically to match the ambient gas pressure at the current location of the bubble.

Of course the particular shape of the torus/mushroom structure (e.g. two tori at different distances from the center in Fig.3) is the result of our choice of initial and boundary conditions and assumed axial symmetry. For example, Fig. 4 shows snapshots of the temperature distribution for slightly different initial conditions (with an initial temperature contrast, between the ambient gas and the bubble, which is a factor of two larger than in the previous run). Note the changes in the detailed morphology of the rising bubble compared to Fig.3. In general one would expect a large variety of structures to be formed, especially in $3 \mathrm{D}$. But the torus-like geometry is a generic feature 


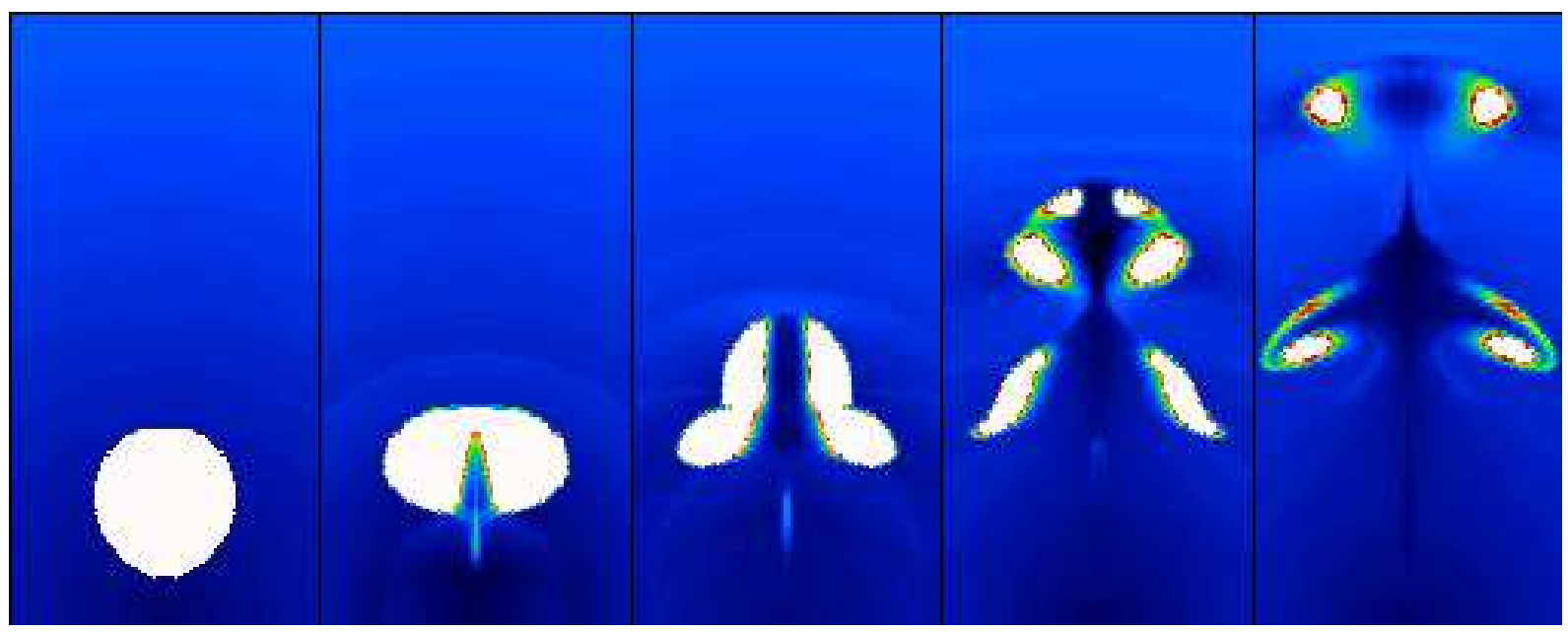

Fig. 3.- Temperature distribution in the gas at 5 time steps. From left to right: 0, 8.4, 21, 42 and 67 Myrs after the start of the simulation. Each box is 40 by $20 \mathrm{kpc}$. The center of the cluster is at the bottom of the box. Temperature is color coded in the 0.7 (black) to $5 \mathrm{keV}$ (white) range. The temperature of the cluster thermal gas (blue) changes from $\sim 1 \mathrm{keV}$ at the center to $\sim 1.7 \mathrm{keV}$ at a distance of $40 \mathrm{kpc}$. All temperatures above $5 \mathrm{keV}$ are white. Thus, the hot "radio-emitting plasma" which initially has a temperature of order 100 $\mathrm{keV}$ is white. Numerical diffusion (due to very strong gradients in density and temperature) creates regions with intermediate values of temperature (yellow, red, green) along the boundaries of the bubble. Note that during later stages, the coldest gas is not at the centre of the cooling flow, but is associated with the rising bubble. Since radiative cooling was not included in the simulations these cold regions represent uplifted (and adiabatically expanded) gas.

of rising bubbles in the absence of strong surface tension.

\subsection{The velocity of the rising bubble}

The simplest velocity estimate of the rising bubble can be obtained by equating the ram pressure and buoyancy forces acting upon a bubble (e.g. Gull and Northover 1973). The buoyancy force is obviously:

$$
F_{\mathrm{b}}=V g\left(\rho_{\mathrm{a}}-\rho_{\mathrm{b}}\right),
$$

where $V$ is the bubble volume, $g$ is the gravitational acceleration (we assume the ambient gas is in hydrostatic equilibrium), $\rho_{\mathrm{a}}$ and $\rho_{\mathrm{b}}$ are the mass densities of the ambient and the bubble gas respectively. The ram pressure (drag) is:

$$
F_{d} \sim C \frac{1}{2} S v^{2} \rho_{\mathrm{a}},
$$

where $S$ is the cross section of the bubble. The numerical coefficient $C$ (drag coefficient) depends on the geometry of the bubble and the Reynolds number. Thus the terminal velocity of the bubble is

$$
v \sim \sqrt{g \frac{V}{S} \frac{2}{C} \frac{\rho_{\mathrm{a}}-\rho_{\mathrm{b}}}{\rho_{\mathrm{a}}}} \sim \sqrt{g \frac{V}{S} \frac{2}{C}} .
$$

Here the factor of $\left(\rho_{\mathrm{a}}-\rho_{\mathrm{b}}\right) / \rho_{\mathrm{a}}$ can be dropped if the bubble density is low compared to the ambient gas density. The expression for the terminal velocity can be further rewritten using the Keplerian velocity at a given distance from the cluster center: $v \sim \sqrt{(r / R)(8 / 3 C)} v_{\mathrm{K}}$, where $r$ is the bubble radius, $R$ is the distance from the center and $v_{\mathrm{K}}=\sqrt{g R}$ is the Keplerian velocity. For a solid sphere moving through an incompressible fluid the drag coefficient $C$ is of the order $0.4-0.5$, for Reynolds numbers in the range $\sim 10^{3}-10^{5}$ (e.g. Landau and Lifshitz 1963). The drag coefficient for a rising bubble should of course be different. First of all, it is not a solid sphere, but a rather complicated structure, resembling a smoke ring in air. Such rings may travel large distances through the air with low drag. Secondly the Mach number 


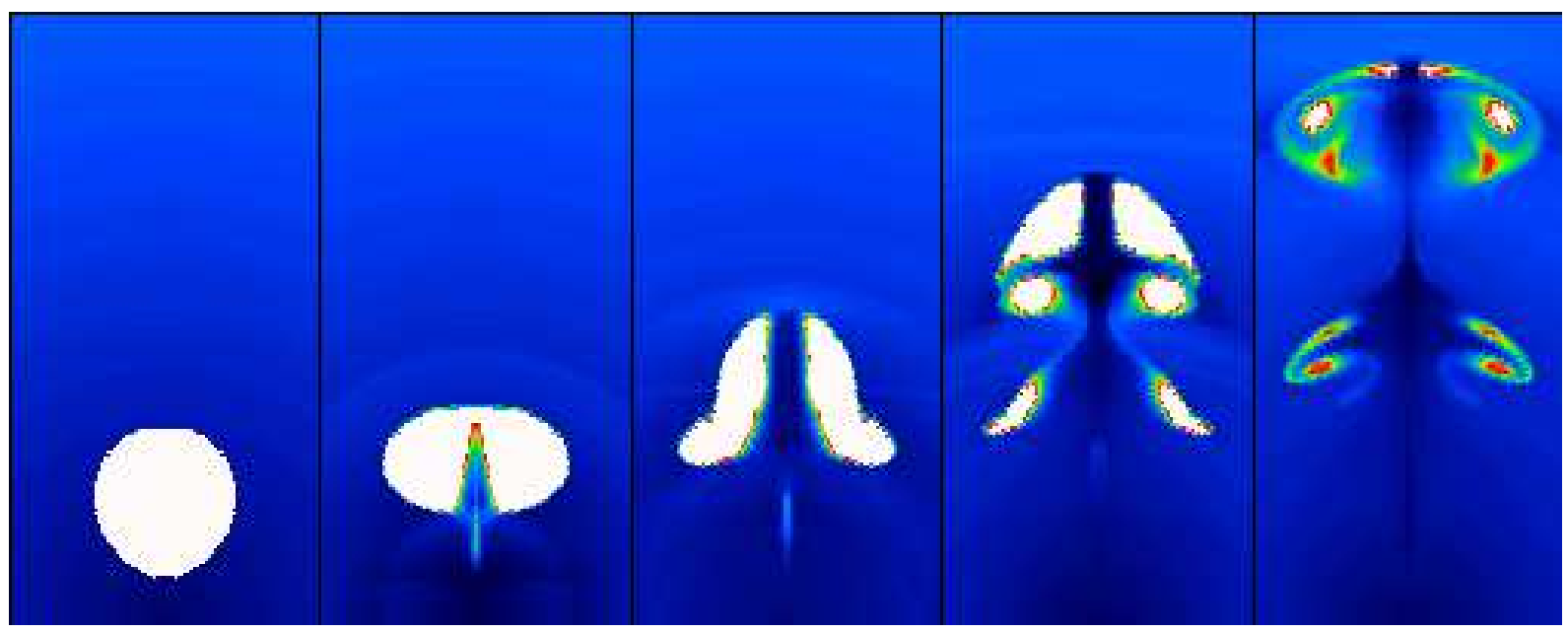

Fig. 4.- The same as in the previous figure but with an initial temperature contrast, between the ambient gas and the bubble, which is a factor of two larger. Note that detailed morphology of the rising bubble is rather sensitive to the initial conditions (and boundary conditions, symmetry assumed etc. ) and can be very different from what is shown in these figures. The presence of the torus-like structure is, however, a generic feature of the rising bubble phenomenon.

of the rising bubble in our simulations is $\sim 0.6-0.7$ and the compressibility of the gas is important. This would tend to increase the drag coefficient, e.g., for a sphere moving at $M \sim 0.7$ the drag coefficient is $C \sim 0.6$. Thirdly, in a stratified medium an additional contribution to the drag may come from the excitation of internal gravity waves. In our simulations, the typical Keplerian velocity was $\sim 400 \mathrm{~km} \mathrm{~s}^{-1}$. Fig.5 shows the position of the bubble front as a function of time. The solid line shows the expected position of the feature moving with the constant velocity of $\sim 390 \mathrm{~km} \mathrm{~s}^{-1}$. The effective drag coefficient which can be estimated from the simulations, assuming the above formula for the rise velocity, is $C \sim 0.75$. We note here that this value may in turn be affected by the numerical resolution adopted here. However, since the velocity of the bubble depends on the square root of the drag coefficient, formula (9) still yields crude order of magnitude estimates of the bubble velocity. Thus, a large and strongly underdense bubble will rise with a velocity comparable to the Keplerian velocity.

In the case of M87, the bubble's environment may not be very underdense compared to the ambient gas (since no clear X-ray holes have been seen) and the velocity of the bubble may be somewhat smaller. However, we believe that the above estimate of the velocity is approximately correct since the velocity depends only weakly (as the 0.5 power) on the bubble size and gas density gradients. This velocity defines a time scale of $\sim$ few $10^{7}$ years for the evolution of the bubble to the stage seen as a bright torus in Fig.1.

\subsection{The rising bubble in synchrotron emission}

In Figure 6 we show the simulation results at $327 \mathrm{MHz}$. The radio emission was calculated for the hydrodynamic simulations shown in Fig.3. Upper and lower rows correspond to different viewing angles. The symmetry axis is perpendicular to the line of sight in the upper row (case 1) in this figure and at an angle of $45^{\circ}$ to the line of sight (case 2) in the lower row. From the radio observations of M87 we note that the well-defined eastern bubble is observed at a distance of roughly $25 \mathrm{kpc}$ from the source centre. This corresponds to the third panel in the upper row of Figure 6, i.e. bubble age of 42 Myrs, while for the simulation projected at an angle of $45^{\circ}$ (lower row), the fourth panel, at an age of 67 Myrs, gives the correct projected distance. Note here that these distances are for the primary bubble/vortex ring. Figure 6 clearly shows the 


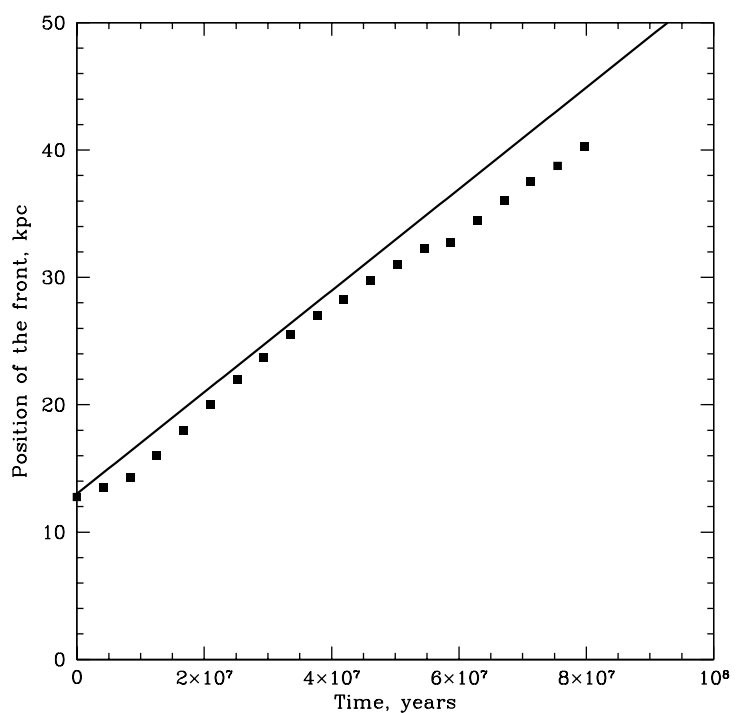

Fig. 5.- Position of the bubble front as a function of time. For comparison solid line shows the motion with the constant velocity of $390 \mathrm{~km} / \mathrm{s}$.

presence of a secondary vortex ring which is closer to the centre. The appearance of this secondary ring is fairly dependent on the particular choice of initial conditions (see Section 2 and Figures 3,4). In the case studied here, the synchrotron surface brightness of the secondary vortex ring exceeds that of the primary bubble. No such structure is observed in M87 and so we concentrate in the following discussion on the primary bubble.

For an initial power law distribution of relativistic electrons in a constant magnetic field, a significant steepening of the synchrotron spectrum will be observed for frequencies higher than the break frequency, $\nu_{\text {break, }}$ which is given by (e.g. Leahy 1991)

$$
\nu_{\text {break }}=2.52 \times 10^{6} \frac{B}{\left(B^{2}+B_{\mathrm{CMB}}^{2}\right)^{2} t^{2}} \mathrm{GHz},
$$

where $B$ is the strength of the magnetic field in $\mu \mathrm{G}$ and $t$ is the time (in Myrs) for which the plasma was exposed to this field. $B_{\mathrm{CMB}}$ is the strength of the magnetic field in $\mu \mathrm{G}$ equivalent to the energy density of the CMB, $u_{\mathrm{IC}}$. For the redshift of M87, $u_{\mathrm{IC}} \sim 4.2 \times 10^{-13} \mathrm{erg} \mathrm{cm}^{-3}$ and so
$B_{\mathrm{CMB}} \sim 3.2 \mu \mathrm{G}$. From equation 10 the time $t$ can be expressed as a function of the break frequency and the strength of the magnetic field. For any given break frequency, the time $t$ is maximal if $B\left(t_{\text {max }}\right)=B_{\mathrm{CMB}} / \sqrt{3} \sim 2 \mu \mathrm{G}$. We have seen that the observed radio spectral indices (Section 1.1) require $\nu_{\text {break }} \sim 3 \mathrm{GHz}$, for which a maximum age is $t_{\max } \sim 10^{8}$ years. This is comparable with the time span of our simulations. For example, if the symmetry axis is at angle of $45^{\circ}$ with the line of sight (case 2 above), then the front of the bubble will reach a projected distance of $\sim 25 \mathrm{kpc}$ after 67 Myrs. This simple estimate shows that we require a rather low value of the initial magnetic field strength (comparable to $B\left(t_{\max }\right) \sim 2 \mu \mathrm{G}$ ) in order to avoid having the $\nu_{\text {break }}$ lie at a frequency lower than $\sim 3 \mathrm{GHz}$.

For case 1 , we used $p=2.3$ and an initial magnetic field strength of $6.5 \mu \mathrm{G}$. We then calculated the synchrotron emission of the bubble as described in Section 2.2. After $\sim 42$ Myrs, i.e. when the bubble is $\sim 25 \mathrm{kpc}$ away from the center, the spectral index of the primary bubble is -2.7 from $4.7-10.6 \mathrm{GHz}$ and -1.0 from $330-$ $1500 \mathrm{MHz}$ which compares well with the observed values (Rottmann et al. 1996, Section 1.1).

For case 2 the magnetic field inside the bubble must be lower since here the bubble is older when its upper edge reaches the correct projected height from the cluster centre. We set $B=2.9 \mu \mathrm{G}$ and $p=2.3$. For this case the high frequency spectral index is found to be -3.0 while at lower frequencies it is -1.0 .

The assumption of pressure equilibrium determines the initial energy density of the relativistic particles. The energy density of the magnetic field is $1.7 \times 10^{-12} \mathrm{ergs} \mathrm{cm}^{-3}$ for case 1 and $3.4 \times 10^{-13}$ ergs $\mathrm{cm}^{-3}$ for case 2 . This is much lower than the energy density of the thermal cluster gas and the particles energy density is therefore $u_{\mathrm{e}} \sim 3 p_{\mathrm{th}} \sim 4.2 \times 10^{-10}$ ergs $\mathrm{cm}^{-3}$ for both cases discussed. This implies a severe departure from the equipartition condition of $u_{\mathrm{B}} \sim u_{\mathrm{e}}$ often assumed for synchrotron emitting plasmas. The initial volume filling factor of the radio emitting plasma in the initial bubble can then be adjusted to provide the required surface brightness of the final bubble. The peak surface brightness predicted in the region of the primary vortex ring is $55 \mathrm{mJy} /$ pixel in case 1 (see Owen et al. 2000, 


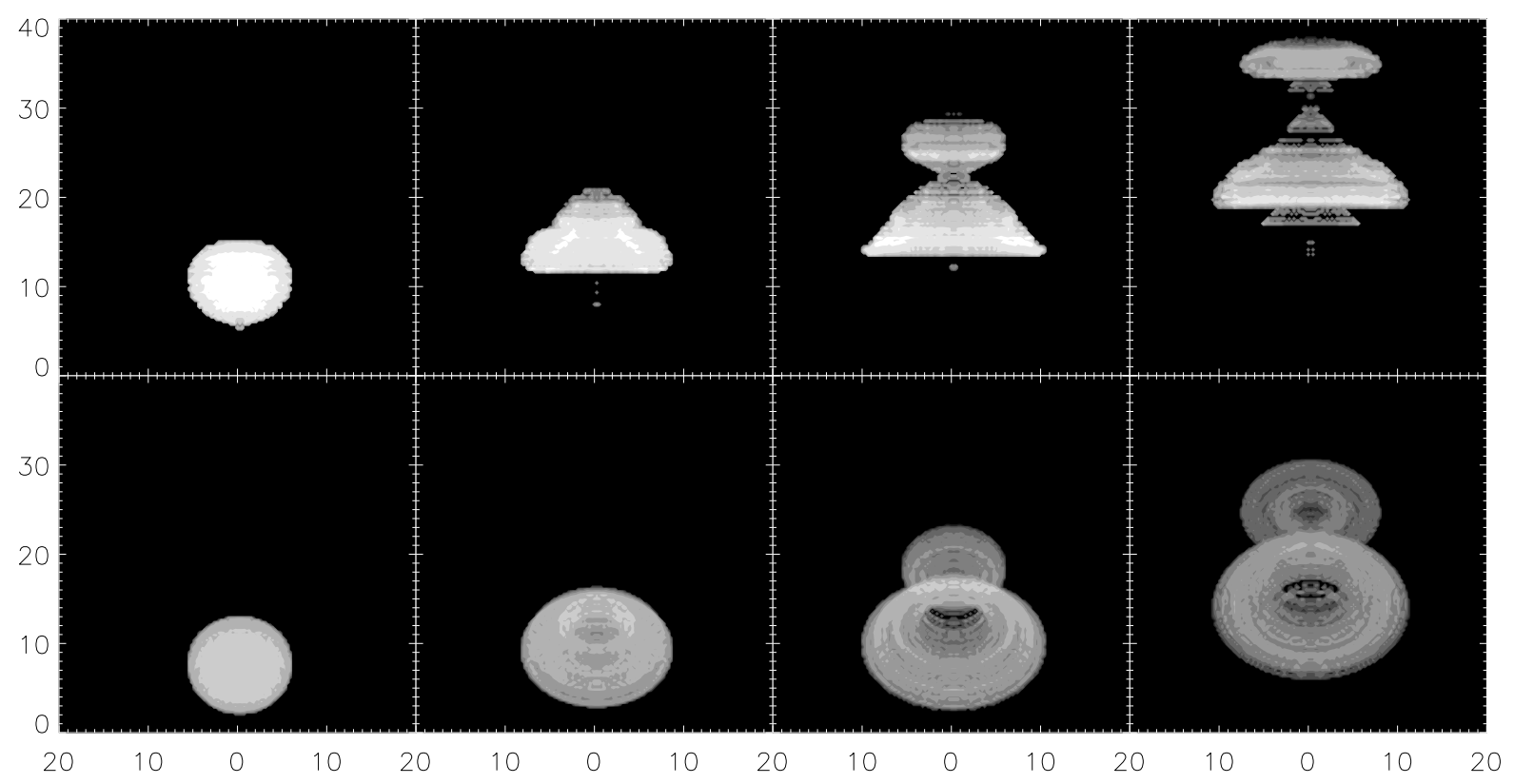

Fig. 6.- Synchrotron surface brightness at $327 \mathrm{MHz}$ at four time steps for the simulation shown in Fig.3. The upper row (case 1) shows the buoyant bubble with a symmetry axis in the plane of the sky and $B \sim 6.5 \mu \mathrm{G}$ initially. The lower row (case 2) is for a symmetry axis inclined $45^{\circ}$ to the line of sight and $B \sim 2.9 \mu \mathrm{G}$ initially. From left to right both rows show the bubble at 4.2, 21, 42 and 67 Myrs after the start of the simulation. The filled contours bound regions of 0.017 (black), 0.04, 0.09, 0.2, 0.45, 1.0, 2.4, 5.5, 13, 28 and 66 (white) mJy per pixel, where a pixel is $250 \times 250 \mathrm{pc}$ at the distance of M87 ( 18 Mpc).

Section 1.1), however, the required volume filling factor of the relativistic plasma is very close to unity. For case 2, even for a filling factor of unity, the peak surface brightness of the bubble is only $2.4 \mathrm{mJy} /$ pixel. Furthermore, we neglected any possible contribution from the relativistic ions (the so-called "k" factor) and thermal plasma to the pressure inside the bubble, which would further reduce its synchrotron emissivity. We also note here that volume filling factors $\sim 1$ pose a serious problem for our assumption that the relativistic fluid does not influence the overall dynamics of the buoyant bubble.

The problems discussed above arise from the life time of the synchrotron emitting particles being short compared to the dynamical time scale of the structure. Gull \& Northover (1973) first noted this lifetime problem for buoyant models. Below, we discuss various solutions.

From Figure 6, we find that the diameter of our primary bubble is smaller than the observed diameter of the eastern bubble in M87. As we showed in Section 3.2, the rise time of the bubble is correlated with its size. It is therefore quite possible that the eastern bubble in M87 is somewhat younger than our simulations suggest. Such a younger age would allow a stronger magnetic field in the bubble and could explain the observed spectrum, which in turn would allow a smaller volume filling factor for the relativistic fluid.

The buoyant bubble may also be filled mainly by a weak magnetic field interspersed with regions of a much higher field strength but small volume filling factor. The relativistic electrons may then survive for a much longer time in the mainly weak magnetic field and only diffuse slowly into the regions of greater field strengths which are responsible for most of the observed synchrotron emission (Eilek, Melrose \& Walker 1997). Subsonic turbulence can also enhance the energy density of the magnetic field in regions with initially weak field (e.g. Eilek, Owen and Zhou 1999). 
If the magnetic field in the buoyant bubbles is stronger than that estimated above and rather homogeneous, then in situ acceleration of relativistic particles is required. In this case, the age of the buoyant bubble cannot be constrained from radio observations. The two latter mechanisms were also discussed by Owen et al. (2000) to explain the radio emission of the outer M87 halo.

The fundamental conclusion of these simulations is that one can reproduce the observed radio brightness and the break in the spectrum of the torus-like feature in M87 assuming that (i) the bubble, filled with the relativistic plasma, is in pressure equilibrium with the thermal gas, (ii) the initial magnetic field strength is relatively low so as to prolong the lifetime of the electrons and (iii) no in situ reacceleration of particles or generation of magnetic field occurs during the bubble evolution. However, the equipartition condition is sacrificed and the parameter space which satisfies these assumptions is rather tight. It is likely that the real situation is much more complex than was assumed in the simple model discussed above.

\subsection{The rising bubble in $\mathrm{X}$-ray emission}

As is clear from Fig.3, the bubble captures ambient gas during its transformation to a torus and then uplifts this gas to larger radii. Since all motions in our simulations are subsonic, this uplifted gas remains in approximate pressure equilibrium with the ambient gas at larger radii. The volume emissivity of this gas can be compared with the volume emissivity of the ambient gas:

$$
\begin{aligned}
\epsilon_{u} & =n_{u}^{2} \Lambda\left(T_{u}\right) \\
\epsilon_{a} & =n_{a}^{2} \Lambda\left(T_{a}\right),
\end{aligned}
$$

where $n$ and $T$ are the density and temperature of the uplifted and ambient gas respectively, $\Lambda(T)$ is the emissivity in a given energy band. If we assume adiabatic evolution of the entrained and uplifted gas then:

$$
\begin{gathered}
n_{u}=n_{a, 0}\left(\frac{P_{a}}{P_{a, 0}}\right)^{1 / \Gamma}=n_{a} \frac{T_{a}}{T_{a, 0}}\left(\frac{P_{a}}{P_{a, 0}}\right)^{1 / \Gamma-1} \\
T_{u}=T_{a, 0}\left(\frac{P_{a}}{P_{a, 0}}\right)^{1-1 / \Gamma}
\end{gathered}
$$

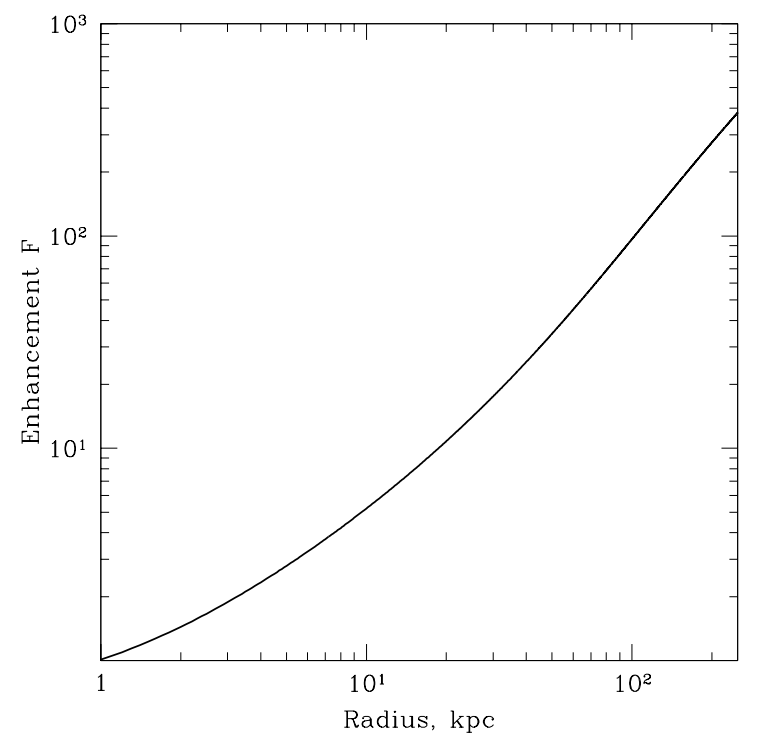

Fig. 7.- Volume emissivity of the uplifted and adiabatically expanded gas relative to the ambient gas. The curve is normalized to unity at 1 kpc. The enhancement factor, $F(r)$, is the ratio of the emissivity of the gas uplifted from $1 \mathrm{kpc}$ to $r$ to that of the ambient gas at $r$. The ratio $F\left(r_{2}\right) / F\left(r_{1}\right)$ characterizes the factor by which volume emissivity of the gas uplifted from $r_{1}$ to $r_{2}$ exceeds the volume emissivity of the ambient gas at $r_{2}$.

where the subscript 0 stands for the values of the uplifted gas at its initial location where it was captured by the rising bubble. Thus the uplifted gas will have an emissivity which is higher than the ambient gas by a factor:

$$
F=\frac{\epsilon_{u}}{\epsilon_{a}}=\left(\frac{T_{a}}{T_{a, 0}}\right)^{2}\left(\frac{P_{a}}{P_{a, 0}}\right)^{2 / \Gamma-2} \times \frac{\Lambda\left(T_{u}\right)}{\Lambda\left(T_{a}\right)} .
$$

The enhancement factor $F$ (excluding the cooling functions) is shown in Fig.7. One can see that in a cooling flow (density decreases with distance while temperature increases), the emissivity of the uplifted gas greatly exceeds the emissivity of the ambient gas if the displacement is large. The ratio $\Lambda\left(T_{u}\right) / \Lambda\left(T_{a}\right)$ (when the temperatures are near 1 $\mathrm{keV}$ and the soft $\mathrm{X}$-ray band is considered) further increases the brightness of the uplifted gas with respect to the ambient gas. 


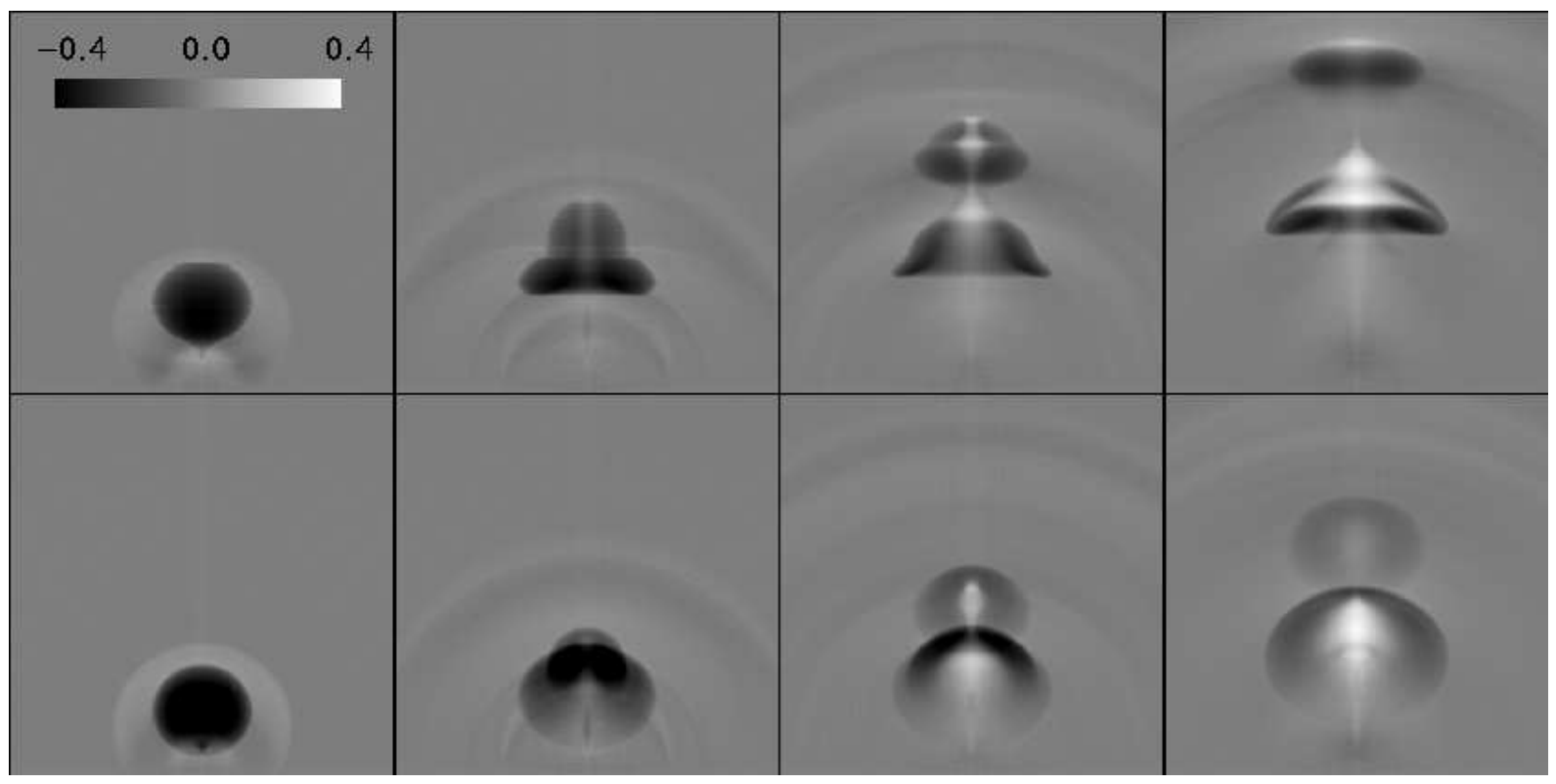

Fig. 8. - Expected X-ray morphology of the bubble for the same times as in Fig.6. Each box is 40 by $40 \mathrm{kpc}$. The figure shows the relative deviation of the X-ray surface brightness (in the ROSAT energy band) with respect to the unperturbed $\mathrm{X}$-ray emission of the cooling flow. The darker regions are $\mathrm{X}$-ray underluminous and lighter regions are X-ray bright. Black corresponds to regions dimmer by $40 \%$ (or more) than the unperturbed value and white corresponds to regions brighter by $40 \%$ (or more) than the unperturbed value.

To illustrate how the $\mathrm{X}$-ray surface brightness is affected by the a radio emitting plasma bubble and lumps of uplifted cold gas, we calculated the projected surface brightness distribution (for the ROSAT energy band from 0.5 to $2 \mathrm{keV}$ ) for several stages of the bubble evolution. When calculating these maps, we assumed axial symmetry and assumed that the axis of the bubble is either perpendicular to the line of sight (upper row of images in Fig.8) or that the angle between the line of sight and bubble axis is $45^{\circ}$ (lower row of images in Fig.8). Fig.8 shows the relative deviation of the $\mathrm{X}$-ray surface brightness from the unperturbed value of the surface brightness at the same distance from the center. As expected the "trunk" of the mushroom is brighter than the surrounding regions.

Fig.9 illustrates the effect of entrainment of cold gas as the bubble rises. Shown in this figure are the distributions of tracer particles at the initial and final (after $\sim 67 \mathrm{Myrs}$ ) stages of the bubble evolution. The tracer particles fill the entire volume outside the bubble. The color assigned to each particle characterizes its initial distance from the cluster center. For example, "green" and "blue" particles seen in the upper torus have been uplifted from the central region of the galaxy or cluster atmosphere.

Abundance gradients are frequently observed in cluster atmospheres around central bright galaxies (e.g. Matsumoto et al. 1996). If our interpretation of the X-ray features as uplifted lumps of gas is correct, then one would expect the abundance in these regions to be higher than in the surrounding regions. This can be tested with Chandra and XMM.

\subsection{The pancake stage}

If the lumps of radio and X-ray emitting plasmas in the bubble are bound together (e.g. by magnetic stresses as in the multiphase cooling flow picture of Nulsen (1986)) then one can calculate the maximum radius to which the whole bubble can rise for given volume fractions of "entrained" gas and (weightless) radio emitting plasma (see eq. (6) in Churazov et al. 2000). Fig.10 shows 


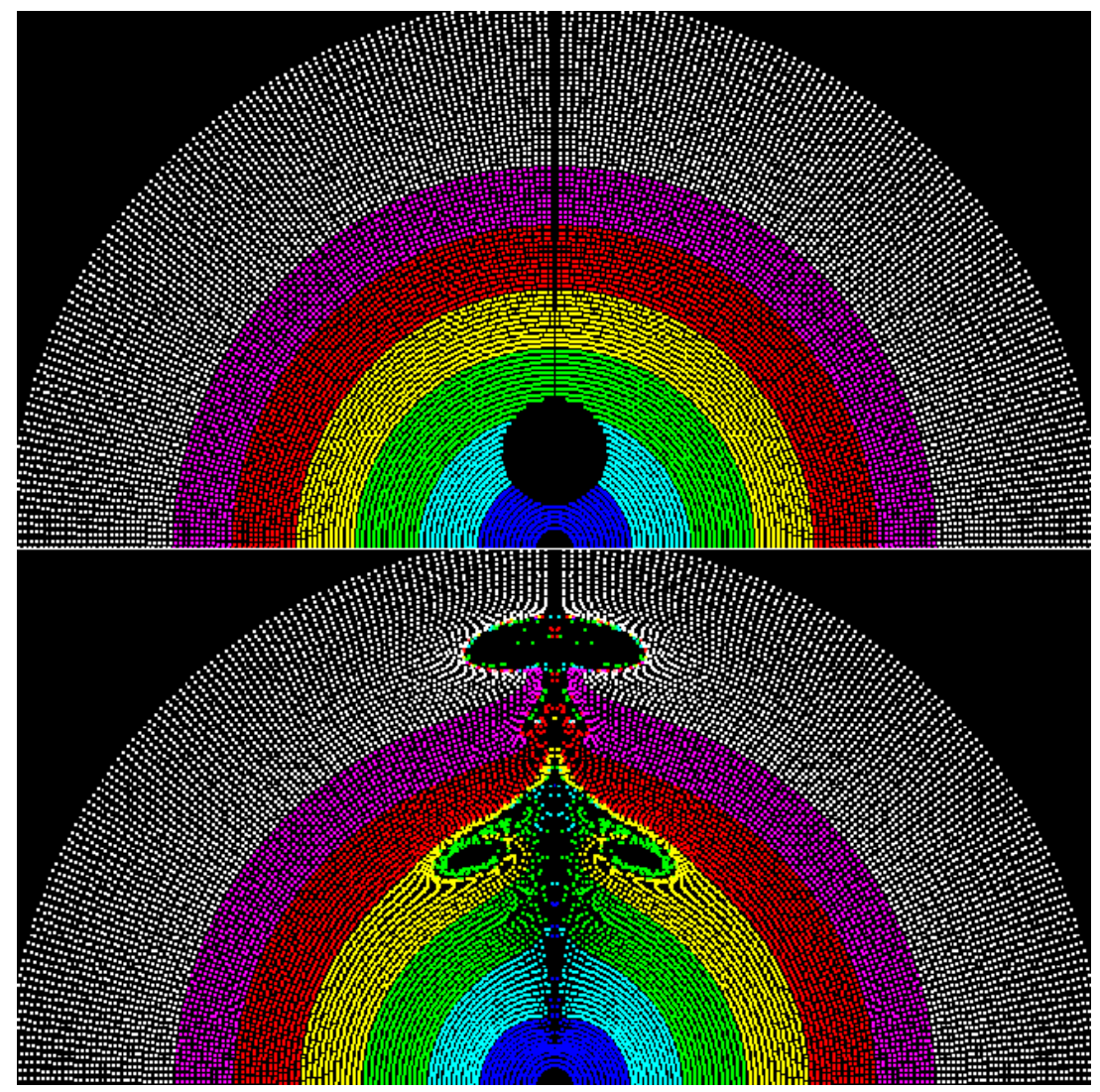

Fig. 9.- Initial (top) and final (bottom) positions of tracer particles, originating within different regions of the cluster gas. Each particle is color coded according to its initial distance from the center. Bottom panel shows the positions of particles 67 Myrs after the beginning of the simulation. "Green" and "blue" particles seen in the upper torus (bottom panel) have been uplifted from the central region of the cooling flow.

the dependence of the final position of the bubble on the initial position and fraction of the bubble volume occupied by entrained gas which was captured near the initial location of the bubble. At this final location, the average mass density of the bubble (consisting of separate lumps of radio and $\mathrm{X}$-ray emitting plasma) is equal to the density of the ambient gas. At the final position the bubble will spread along the equipotential (isodensity) surface and may form a pancake-like structure. The $\mathrm{X}$-ray appearance of the bubble at this stage crucially depends on whether mixing of radio and $\mathrm{X}$-ray emitting plasmas is microscopic or macroscopic (Böhringer et al. 1995, Nulsen 1997)

A simple simulation demonstrating pancake formation is presented in Fig.11. In this simulation, we assumed that the bubble is initially filled with gas having the same entropy as the cluster gas at a distance of $\sim 25 \mathrm{kpc}$. This is done by setting the temperature of the gas inside the bubble a factor of $\sim 2$ higher than the temperature of the surrounding cluster gas. The density in the bubble was set a factor of 2 lower in order to maintain pressure equilibrium. The resulting modest contrast in entropy between the bubble and the surrounding gas approximately models the situation when the filling factors of the radio emitting plasma and thermal plasma in the bubble are comparable as considered in the previous section. The upper row of Fig.11 shows the entropy distribution at the beginning of the simulation and after $\sim 2.6 \times 10^{8}$ years. In the lower panel, we show the distribution of tracer particles, which initially are located within the bubble, at the same times. The 


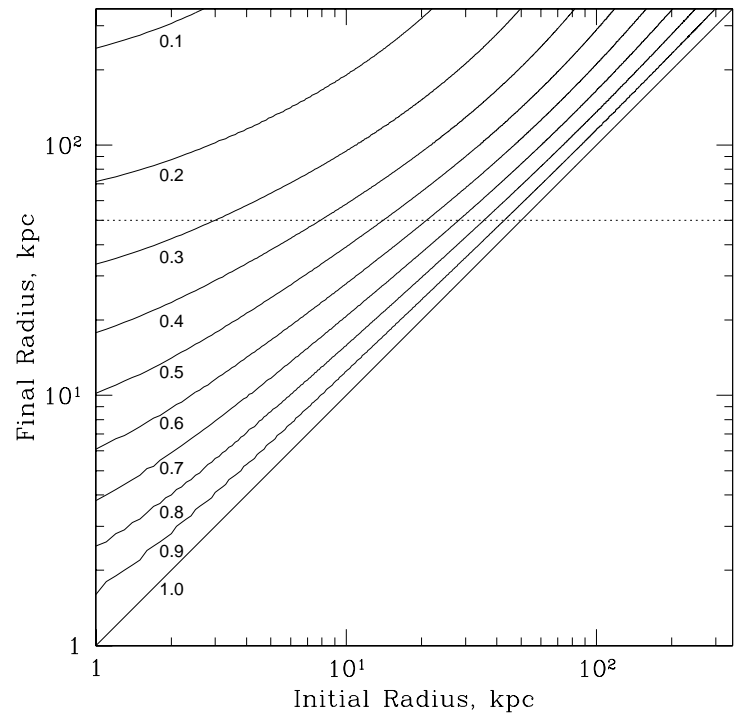

Fig. 10. - The maximum radius to which the bubble can rise depending on its initial location and the volume fraction of gas, captured during the transformation of the bubble into a torus. It is assumed that the bubble consists of separate lumps of radio and $\mathrm{X}$-ray emitting plasmas bound together. Each curve corresponds to a given volume fraction (labels under the curves) of entrained gas added to the bubble near the original bubble position. If, for example, the large circular radio emission regions are located at a distance of 50 $\mathrm{kpc}$ from the center (horizontal dotted line) then a bubble could rise from $5 \mathrm{kpc}$ to $50 \mathrm{kpc}$ with the amount of "entrained" gas corresponding to approximately $35 \%$ of its initial volume. two black curves show, respectively, the boundary of the bubble at the beginning of the simulation and the region in the cluster where the cluster gas has the same entropy as the gas initially inside the bubble. It is clear that at the end of the simulation, tracer particles are concentrated along a surface where cluster gas has a similar entropy to that of the gas in the original bubble. Pancakelike structures are already visible after $\sim 10^{8}$ years from the start of the simulations. Oscillations near the neutral buoyancy point were also observed.

Strongly underdense bubbles may rise to much larger distances from the cluster center before reaching a neutral buoyancy point (see Fig.10). We did not simulate the evolution of strongly underdense bubbles to such late stages, but qualitatively the picture should be the same.

The largest structures visible in Figure 1 are the two almost circular low surface brightness emission regions to the northeast and southwest of the centre. Owen et al (2000) suggest that these are spherical bubbles of radio plasma seen in projection. In this case, they may receive substantial energy input from the inner lobes which contain the active jet. Alternatively one can identify the circular emission regions seen in the radio images with earlier bubbles that have reached their isodensity distance and are now in the process of spreading and forming pancake-like structures. We stress again that this requires that either relativistic and thermal particles are mixed on microscopic scales or the lumps of relativistic plasma and the ambient medium are bound together.

Owen et al. (2000) have noted the sharp boundary of the M87 halo which is traced by all observations at different frequencies and they argue that this excludes a dominant diffusive transport process for the relativistic plasma. In the proposed model the radius and sharpness of the boundary is naturally explained by the height to which buoyant bubbles can rise. Also the complicated structure of the outer halo may in this model reflect several layers of partly fragmented bubbles.

The time required for pancake formation at large distances from the cluster center is at least several times longer than that required for a strongly buoyant bubble to travel such distances. Therefore, it is extremely difficult for relativistic particles to survive (see 3.3). Either reacceleration or exposure of relativistic particles to stronger 
magnetic fields seems to be inevitable to explain the radio emission from the outer radio halo if it is indeed the result of lateral spreading of a bubble which has reached the neutral buoyancy point.

If, on the contrary, lumps of radio emitting plasma are not bound to the captured lumps of thermal gas, then radio bubbles will continue to rise along the pressure gradient and will eventually leave the central cooling flow region. Overdense lumps of captured gas will separate from the bubbles and fall back towards the central region. One possible example of overdense material having fallen back toward the central galaxy may be found in A1795. Cowie et al. (1983) reported a remarkable $\sim 45^{\prime \prime}$ (41 kpc long for the Hubble constant of $100 \mathrm{~km} \mathrm{~s}^{-1} \mathrm{Mpc}^{-1}$ ) filamentary emission line $(\mathrm{H} \alpha+[\mathrm{NII}])$ chain originating near the central $\mathrm{cD}$ galaxy.

\subsection{Total energy budget}

The X-ray luminosity of the whole cooling flow region around M87 is about $10^{43} \mathrm{erg} \mathrm{s}^{-1}$. On the other hand, estimates of the total energy supplied by the jet to the ICM give a much larger value of $\sim 10^{44} \mathrm{erg} \mathrm{s}^{-1}$ (e.g. Owen, Eilek and Kassim 2000). If all this energy can be dissipated into the thermal gas in the cooling flow region then the cooling flow should instead be a "heating" outflow (Owen, Eilek and Kassim 2000), at least at the present moment. The situation may be transient so that inflow and outflow periods alternate depending on the strength of the AGN activity (e.g. Tucker and David 1997, Binney 1999, Owen, Eilek and Kassim 2000). We speculate below on the consequences of a large input of power in the form of relativistic plasma into the cooling flow region.

Initially the radio lobe, inflated by the jet, expands supersonically (e.g. Heinz, Reynolds, Begelman 1998). Later, as the size of the radio lobe grows, the expansion becomes subsonic. As we discussed above (Section 3.2), a large and strongly underdense bubble would rise with approximately the Keplerian velocity of $\sim 400 \mathrm{~km} \mathrm{~s}^{-1}$. Assuming that this velocity limits the growth of the original bubble due to the jet input power $L$, one can write: $\frac{\Gamma}{\Gamma-1} P_{0} 4 \pi r^{2} v=L$, where $P_{0}$ is the ambient gas pressure, $\Gamma=4 / 3$ is the adiabatic index of the relativistic gas in the bubble, $r$ is the size of the bubble. Substituting $L \sim 10^{44} \mathrm{erg} \mathrm{s}^{-1}$, $P_{0} \sim 2 \times 10^{-10} \mathrm{erg} \mathrm{cm}^{-3}$ and $v \sim 400 \mathrm{~km} \mathrm{~s}^{-1}$ we find $r \sim 5 \mathrm{kpc}$, which is approximately the size of the inner lobes surrounding the jets. If the subsonic expansion phase is longer than the duration of the initial strongly supersonic expansion, then most of the energy deposited by the AGN will go into the enthalpy of the expanded lobe and not into shock heating the cluster gas.

The inner lobes break loose (during a time $t \sim r / v \sim 10^{7}$ years) and form bubbles which then subsonically rise through the cluster atmosphere (Gull and Northover 1973). At any given moment, thermal gas tries to settle in the potential of the cluster in order to form a nondecreasing entropy profile. Rising bubbles produce convective motions in the gas and mix lumps of thermal gas with different entropies. Using tracer particles added to the thermal gas (see Fig.9) we estimated that some $3-4 \times 10^{7} M_{\odot}$ of cool, thermal gas has been uplifted from below $15 \mathrm{kpc}$ to above $35 \mathrm{kpc}$ by the bubble after $\sim 8 \times 10^{7}$ years. Much larger masses of gas undergo smaller displacements by that time. For example, $\sim 1.3 \times 10^{8} M_{\odot}$ has been lifted from below $14 \mathrm{kpc}$ to above $25 \mathrm{kpc}$. Although these are very crude estimates, which are sensitive to the particular initial and boundary conditions adopted in our simulations, one can expect that if the bubbles are launched approximately every $10^{7}$ years then they are able to transport some $1-10 M_{\odot}$ of cold gas per year from the center to the periphery of the cooling flow. Interestingly this value is comparable to the mass deposition rate in M87 estimated from the X-ray data. One can further list several important consequences of the convection induced by the rising bubbles:

- Due to uplifted cold gas, the X-ray surface brightness distribution should be shallower than in the absence of bubbles. The entropy profiles also flatten.

- If the bubbles are formed and are rising systematically over some preferred direction then the cold gas will be continuously uplifted over this direction while it will be flowing inward in other directions.

- Thermal instabilities can effectively take place within an environment of extensive convective motions. As a result, distributed mass deposition from cooling gas can naturally occur. 
- Overdense lumps of thermal gas, uplifted by the rising bubbles, may fall back (when separated from the "carrier" buoyant bubble) to the central region thereby producing filamentary structures, stretching radially from the galaxy.

Even if the bubbles are not mixed on microscopic scales with the thermal plasma and are able to eventually leave the cooling flow region, most of the energy has to be deposited in the thermal gas (if there is a strong contrast in pressure between the inner and outer regions of the cooling flow). For a bubble moving at a constant terminal velocity the dissipation of energy is characterized by the change of the bubble enthalpy $\frac{\Gamma}{\Gamma-1} P V \propto\left(P / P_{0}\right)^{\frac{\Gamma-1}{\Gamma}}$, where $P$ is the pressure of the cluster thermal gas. The bubble energy goes into sound waves, internal waves, turbulent motion in the wake, potential energy of the uplifted cold gas etc. Sound waves can carry energy away from the central region, while most of the other channels would eventually result in heating the cooling flow region. If the jet power is indeed $\sim 10^{44} \mathrm{erg} \mathrm{s}^{-1}$, then even a modest $10 \%$ efficiency of local dissipation into heat should be enough to exceed the radiative cooling of the gas. The gas then heats up, but this heating is distributed through a large volume. Since no shock waves are envisaged in this picture one would not expect to find localized, hot, compressed regions.

If strong energy input from AGN is maintained for a very long period (and dissipation into heat is efficient), then a core with a flat entropy profile will be formed and the size of this core will grow with time. We note here that the rate of energy extraction from the bubble depends on the pressure gradient (see above expression for enthalpy). Therefore, if the gas is heated to a temperature comparable to the depth of the local potential well, the dissipation efficiency will decrease in this region. This effect may provide a self-regulation for heating in the central part of the cooling flow region.

Eventually, strong heating is capable of completely eliminating the cooling flow, unless the power of an AGN is in turn regulated by the conditions in the cooling flow region as discussed by Tucker and David (1997), Binney (1999), Owen, Eilek and Kassim (2000). In M87, heating at a rate of $\sim 5 \times 10^{43} \mathrm{ergs} \mathrm{s}^{-1}$ would eliminate the cooling flow for $\sim 10^{8}$ years. This is comparable to the age of the largest radio structures. On the other hand, the complicated radio halo morphology suggests that periods of high AGN activity have alternated with the periods of low AGN power. We note here that dissipation of the subsonic motions into heat may take a longer time than the characteristic bubble rise time and therefore the actual gas heating rate may be related to the AGN activity averaged over a long period of time.

\section{Conclusions}

Buoyant bubbles of cosmic rays, slowly rising through the cooling gas, can qualitatively explain the complicated radio and $\mathrm{X}$-ray morphology of the central $40 \mathrm{kpc}$ region around M87. Toruslike features, seen in the radio, may be similar to the "mushrooms" which appear in RayleighTaylor unstable configurations: as the fluid rises through the ambient medium, Kelvin-Helmholtz instabilities create torus-like "mushroom" heads. The excess $\mathrm{X}$-ray emission trailing some prominent radio features could be due to cold gas captured by the rising bubbles and uplifted to large distances from the central source.

Rising bubbles produce strong convection in the cooling flow region. Convection flattens $\mathrm{X}$ ray surface brightness and entropy profiles in the cooling flow. Convection may promote distributed mass deposition in the cooling flow and the formation of the filaments. If more than $10 \%$ of the energy supplied by the jet is dissipated into heat in the cooling flow region, then large cores with flat entropy profiles may be formed.

New Chandra and XMM observations of cooling flow clusters (e.g. McNamara et al. 2000, Fabian et al. 2000a) demonstrate that the interaction of the radio sources and thermal gas is widespread in these objects. Furthermore, the mass of cooling gas at temperatures less than $\sim 1$ $\mathrm{keV}$, derived from the spectra, seems to be significantly smaller than expected from the simplest cooling flow models (Böhringer et al. 2001, Tamura et al. 2001, Peterson et al. 2001). Possible explanations for this discrepancy are summarized by Fabian et al. (2000b; see also references therein). As we discussed above, heating 

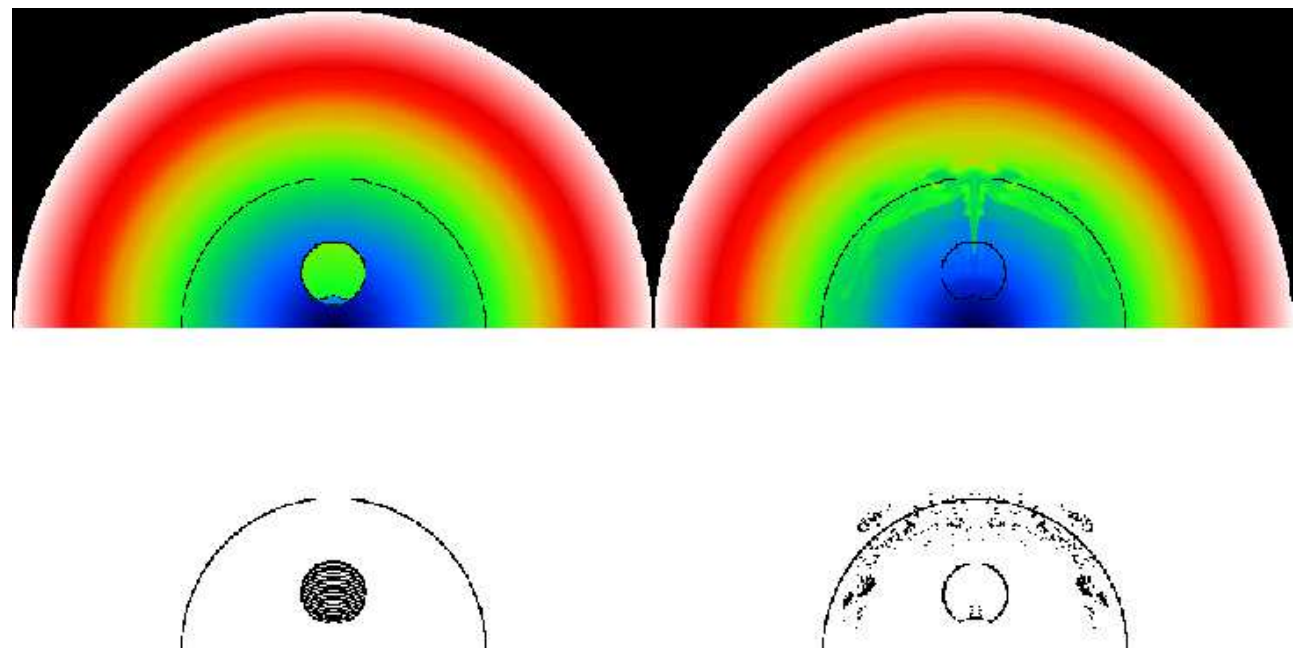

Fig. 11. - Simple simulations demonstrating formation of a pancake. The top panel shows the distribution of the quantity $T / n^{2 / 3}$ which characterizes the entropy of the gas. Bottom panel shows the distribution of tracer particles, originally located within the bubble. The left column corresponds to the state shortly after the beginning of simulations and the right column corresponds to the state after $\sim 2.6 \times 10^{8}$ years. The initial entropy of the bubble (green circle in the top-left plot) was such that the cluster gas has a similar entropy at a radius of $25 \mathrm{kpc}$ from the center. The entropy contrast between the bubble and surrounding cluster gas is rather modest and the bubble travels only a small distance from the center. The contours of the initial bubble and a circle with a $25 \mathrm{kpc}$ radius are shown by black lines. The outer edge of the simulated volume (outer black line in the bottom panel) corresponds to a distance of $50 \mathrm{kpc}$ from the cluster center. It is clear that at the end of the simulation, tracer particles are concentrated along the surface where cluster gas has a similar entropy to that of the gas in the original bubble.

by the buoyant bubbles may play an important role in the thermal balance in cooling flows. One of the predictions of the buoyant bubble picture, namely a correlated morphology of the colder uplifted gas and radio emission, seems to be in a broad agreement with recent XMM results (Belsole et al. 2001).

Our model is of course highly oversimplified and is not intended to closely reproduce the M87 environment. Clearly more detailed simulations are needed, perhaps in 3D, which would also follow the formation of the initial bubble (possibly with continuous energy input) and include radiative cooling. However, despite the simple treatment of bubbles in cluster and galaxy atmospheres presented above, we believe that this approach indicates areas of particular interest.

We are grateful to the editor Steven N. Shore and the referee for important comments and suggestions. We thank Alexei Kritsuk, Nail In- ogamov, Christine Jones, Nail Sibgatullin and Henk Spruit for useful discussions. This research has made use of data obtained through the High Energy Astrophysics Science Archive Research Center Online Service, provided by the NASA/Goddard Space Flight Center.

\section{REFERENCES}

Batchelor, G.K., 1967, An introduction to fluid dynamics, Cambridge University Press

Baum, S. A. \& O'Dea, C. P. 1991, MNRAS, 250, 737

Belsole, E., Sauvageot, J. L., Böhringer, H., Worrall, D. M., Matsushita, K., Mushotzky, R. F., Sakelliou, I., Molendi, S., Ehle, M., Kennea, J., Stewart, G. \& Vestrand, W. T. 2001, A\&A, 365, L188

Binney, J. 1999, Berlin Springer Verlag Lecture Notes in Physics, 530, 116 
Biretta, J. A. 1999, Berlin Springer Verlag Lecture Notes in Physics, 530, 159

Böhringer, H., Voges, W., Fabian, A. C., Edge, A. C. and Neumann, D. M. 1993, MNRAS, 264, L25

Böhringer, H., Nulsen, P. E. J., Braun, R. and Fabian, A. C. 1995, MNRAS, 274, L67

Böhringer, H., 1999, Diffuse Thermal and Relativistic Plasma in Galaxy Clusters, eds. H.Böhringer, L.Feretti, P. Schuecker, MPE Report 271,115

Böhringer, H., Belsole, E., Kennea, J., Matsushita, K., Molendi, S., Worrall, D. M., Mushotzky, R. F., Ehle, M., Guainazzi, M., Sakelliou, I., Stewart, G., Vestrand, W. T. \& Dos Santos, S. 2001, A\&A, 365, L181

Bolton J.G., Stanley G.J., Slee O.B., 1949, Nature, 164, 101

Churazov, E., Forman, W., Jones, C. and Böhringer, H. 2000, A\&A, 356, 788

Cowie, L. L., Hu, E. M., Jenkins, E. B. and York, D. G. 1983, ApJ, 272, 29

Eilek, J. A., Melrose, D. B. and Walker, M. A. 1997, ApJ, 483, 282

Eilek, J., Owen, F. and Zhou, F. 1999, Berlin Springer Verlag Lecture Notes in Physics, 530, 136

Fabian, A. C., Sanders, J. S., Ettori, S., Taylor, G. B., Allen, S. W., Crawford, C. S., Iwasawa, K., Johnstone, R. M. \& Ogle, P. M. 2000a, MNRAS, 318, L65

Fabian A.C., Mushotzky R.F., Nulsen P.E.J., Peterson J.R., 2000b, MNRAS, submitted, astro$\mathrm{ph} / 0010509$

Feigelson, E. D., Wood, P. A. D., Schreier, E. J., Harris, D. E. and Reid, M. J. 1987, ApJ, 312, 101

Gull, S.F., Northover, K. J. E. 1973 Nature, 244, 80

Harris D.E.,Owen F.N.,Biretta J.A., Junor W., 1999, Diffuse Thermal and Relativistic Plasma in Galaxy Clusters, eds. H.Böhringer, L.Feretti, P. Schuecker, MPE Report 271, 111
Heinz, S., Reynolds, C. S. \& Begelman, M. C. 1998, ApJ, 501, 126

Huang, Z. and Sarazin, C. L. 1998, ApJ, 496, 728

Inogamov, N. A. 1999, Astrophysics and Space Physics Reviews, 10, 1

Kaiser C.R., Dennett-Thorpe J., Alexander P., 1997, MNRAS 292, 723

Kassim, N. E., Perley, R. A., Erickson, W. C. and Dwarakanath, K. S. 1993, AJ, 106, 2218

Landau, L.D., Lifshitz, E.M., 1963, Fluid mechanics, Pergamon Press

Leahy J.P., 1991, in P. A. Hughes (ed.), Beams and jets in astrophysics. Cambridge University Press, p. 100

Longair M.S., 1994, High energy astrophysics, Cambridge University Press

Matsumoto, H., Koyama, K., Awaki, H., Tomida, H., Tsuru, T., Mushotzky, R. and Hatsukade, I. 1996, PASJ, 48, 201

McNamara, B. R., O'Connell, R. W. and Sarazin, C. L. 1996, AJ, 112, 91

McNamara, B. R., Wise, M., Nulsen, P. E. J., David, L. P., Sarazin, C. L., Bautz, M., Markevitch, M., Vikhlinin, A., Forman, W. R., Jones, C. and Harris, D. E. 2000, ApJ, 534, L135

Mills B.Y., 1952, Nature, 170, 1063

Norman, M.L., Smarr, L., Winkler, K.-H., Smith, M.D., 1982, A\&A, 113, 285

Nulsen, P. E. J. and Böhringer, H. 1995, MNRAS, 274, 1093

Nulsen, P. E. J. 1986, MNRAS, 221, 377

Nulsen, P. E. J. 1997, ASP Conf. Ser. 115: Galactic Cluster Cooling Flows, 135

Onufriev, A.T. 1967, Zhurnal Prikladnoi Mekhaniki i Tehnicheskoi Fisiki, 2, 101

Owen F.N., Eilek J.A., Kassim N.E., 1999, Diffuse Thermal and Relativistic Plasma in Galaxy Clusters, eds. H.Böhringer, L.Feretti, P. Schuecker, MPE Report 271, 107 
Owen, F. N., Eilek, J. A. \& Kassim, N. E. 2000, ApJ, 543, 611

Peres, C. B., Fabian, A. C., Edge, A. C., Allen, S. W., Johnstone, R. M. and White, D. A. 1998, MNRAS, 298, 416

Peterson, J. R., Paerels, F. B. S., Kaastra, J. S., Arnaud, M., Reiprich, T. H., Fabian, A. C., Mushotzky, R. F., Jernigan, J. G. \& Sakelliou, I. 2001, A\&A, 365, L104

Rottmann H., Mack K.-H., Klein U., Wielebinski R., 1996, A\&A 309, L19

Stone, J.M., Norman, M.L., 1992a, ApJS, 80, 753

Stone, J.M., Norman, M.L., 1992b, ApJS, 80, 791

Tamura, T., Kaastra, J. S., Peterson, J. R., Paerels, F. B. S., Mittaz, J. P. D., Trudolyubov, S. P., Stewart, G., Fabian, A. C., Mushotzky, R. F., Lumb, D. H. \& Ikebe, Y. 2001, A\&A, $365, \mathrm{~L} 87$

Tucker, W. and David, L. P. 1997, ApJ, 484, 602

Turner, J.S., 1973, Buoyancy effects in fluids, Cambridge University Press

van Leer, B. 1977, Journal of Computational Physics, 23, 276

Walters, J.K. Davidson, J.F. 1963, J.Fluid.Mech., 17,321

Zhidov, I.G. Meshkov, E.E. Popov, V.V.Rogachev, V.G. Tolshmyakov A.I. 1977, Zhurnal Prikladnoi Mekhaniki i Tehnicheskoi Fisiki , 3, 75

This 2-column preprint was prepared with the AAS LATEX macros v5.0. 